\title{
Modélisation de l'effet du confinement sur le tourbillon marginal d'une aile-3D
}

\author{
par F. Deniset \& C. Pellone
}

Laboratoire des Ecoulements Géophysiques et Industriels, Grenoble

\section{I —INTRODUCTION}

Les écoulements de liquides à grande vitesse ou soumis à de brutales variations de pression sont souvent le siège de dépressions suffisamment importantes pour qu'une vaporisation puisse se produire localement. Il s'agit du phénomène de cavitation. La cavitation se manifeste par l'apparition de petites bulles ou de grandes cavités qui se forment puis implosent en quelques microsecondes. Elle est la source de nuisances graves telles que le bruit, qui favorise largement la détection acoustique des sousmarins, l'érosion des matériaux et occasionne également des chutes de performances dans les installations hydrauliques ou marines: pompes, vannes, turbines, hélices. Parmi les problèmes posés par la cavitation, celui du tourbillon de bout d'aile, appelé tourbillon marginal, doit être considéré en premier puisque son apparition précède effectivement tous les autres, et qu'il peut être la source de bruits intenses. C'est la raison pour laquelle l'Action Concertée Cavitation (A.C.C.) a fait du tourbillon marginal le thème majeur de son programme scientifique 1992 1996. Cette étude s'inscrit donc dans le cadre de l'Action Concertée Cavitation qui regroupe, sous l'égide de la Direction de la Recherche et de la Technologie, les laboratoires de recherche suivants: le Bassin d'Essais des Carènes (Val-de-Reuil), l'Ecole Navale de Brest, l'Ecole Nationale Supérieure des Techniques avancées (Palaiseau), 1'Institut de Machines Hydrauliques et de Mécanique des Fluides de l'Ecole Polytechnique Fédérale de Lausanne et le Laboratoire des Ecoulements Géophysiques et Industriels (Grenoble).

\section{DORMULATION}

Cette partie est consacrée à la présentation de la méthode utilisée pour modéliser l'écoulement tridimensionnel stationnaire d'un fluide parfait autour de l'aile considérée, placée dans une veine en charge de section rectangulaire. Cette méthode prend comme inconnue le potentiel de perturbation dans le domaine correspondant à l'écoulement tout en fixant sa valeur dans le domaine complémentaire, à savoir l'intérieur de la structure étudiée (problème de type Dirichlet). Les hypothèses retenues (écoulement irrotationnel et stationnaire, fluide parfait et incompressible) permettent d'utiliser une méthode potentielle nécessitant une double distribution de singularités, dite «mixte de Green », sur l'aile et son sillage.

\subsection{Position du problème}

L'aile étudiée est en simple translation par rapport à l'écoulement, l'effet de la rotation de l'hélice étant considéré comme non prédominant en première approche. On définit donc un unique repère relatif $R_{r}=(O, x, y, z)$ lié à l'aile, orthonormé direct et de vecteurs directeurs $\vec{i}, \vec{j}, \vec{k}$, le vecteur $\vec{i}$ étant dirigé vers l'aval de l'écoulement, le vecteur $\vec{j}$ dans la direction verticale et le vecteur $\vec{k}$ dans la direction horizontale transverse à l'écoulement (fig. 1).

L'aile et son sillage sont des surfaces symétriques par rapport au plan $(O, x, y)$. L'avance de l'aile est donnée par le vecteur $-V_{0} \vec{i}$. La vitesse relative $\vec{V}$ d'une particule fluide au point $M$, exprimée dans le repère relatif $R_{r}$ lié à l'aile, est la somme de la vitesse à l'infini amont et de la vitesse de perturbation due à la présence de l'aile et s'écrit :

$$
\vec{v}=\vec{V}_{\infty}+\vec{v}
$$

Le problème, formulé pour la vitesse de perturbation, est abordé dans le cadre d'un milieu infini. Nous nous proposons de le résoudre dans le domaine $T$ extérieur à l'aile et à son sillage. L'écoulement est supposé à grand nombre de Reynolds, ce qui permet de négliger les forces de pesanteur.

Dans le domaine $T$, les caractéristiques du champ de vitesse de perturbation gouvernant l'écoulement sont les suivantes :

- irrotationnalité : $\vec{\nabla} \wedge \vec{v}=\overrightarrow{0}$

- incompressibilité : $\boldsymbol{\nabla} \cdot \vec{v}=0$

D'où le champ de perturbation dérive d'un potentiel $(\vec{v}=\vec{\nabla} \varphi)$, harmonique : $\nabla \varphi=0$ 


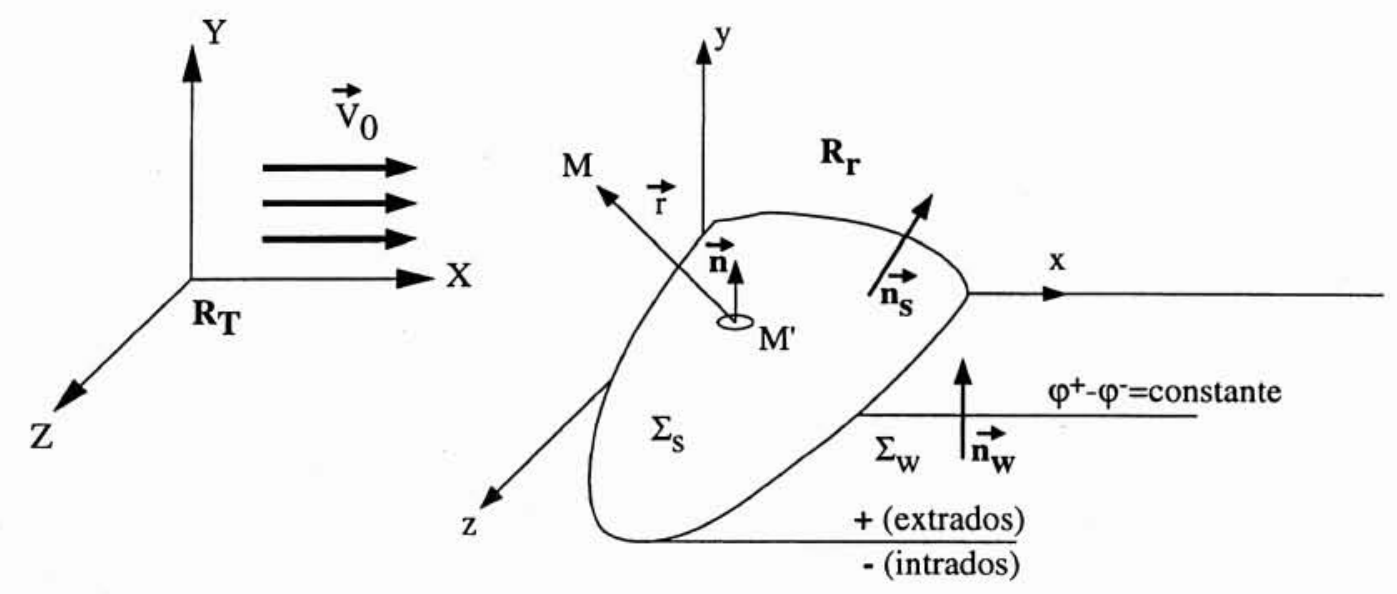

1. Position de l'aile liée au repère relatif $\boldsymbol{R}_{r}$

\subsection{Conditions aux limites}

En écoulement de fluide parfait, la condition de glissement est exprimée sur les surfaces mouillées de l'aile. Il y a également des conditions aux limites sur le sillage, une condition de Joukowski au bord de fuite ainsi qu'une condition à l'infini portant sur le potentiel. Dans le cas général, le sillage est constitué de lignes tourbillons s'échappant du bord de fuite de l'aile en direction de l'aval de l'écoulement, de façon à respecter la conservation de la circulation autour de chaque section. L'ensemble de ces lignes génère une surface de géométrie a priori inconnue mais déterminée par deux conditions :

- la première provient du théorème de Helmoltz qui stipule que le sillage est un domaine matériel. En écoulement stationnaire, cela signifie que ce domaine est une surface de courant ;

- la seconde traduit l'équilibre dynamique du sillage, c'està-dire la continuité du champ de pression à la traversée de la nappe, car cette dernière est une surface non portante.

Ces deux conditions se traduisent en fait par des conditions sur les distributions discrètes de singularités, soit :

$$
\sigma_{w}=0 \text { et } \frac{d}{d t}\left(\mu_{w}\right)=0
$$

où $\sigma_{w}$ et $\mu_{w}$ désignent les distributions respectives de sources et de doublets normaux sur le sillage.

La condition de Joukowski non linéaire exprime l'égalité des pressions de part et d'autre du bord de fuite de l'aile. Aux conditions aux limites précédentes s'ajoutent les conditions à l'infini traduisant la régularité du potentiel de perturbation.

\section{- 2.3 Equations intégrales}

Soient $\Sigma_{s}$ la surface de l'aile, + et - les voisinages du sillage appartenant à $T$, respectivement à l'extrados et à l'intrados et $\Sigma_{w+}$ et $\Sigma_{w-}$, les deux surfaces infiniment proches du sillage réunies en une seule surface $\Sigma_{w}$ de normale $n$ orientée vers l'extrados. L'expression intégrale du potentiel de perturbation est :

$$
\varphi(M)=-\frac{1}{4 \pi} \iint_{\Sigma_{s}}\left[\sigma\left(\frac{1}{r}\right)\right] d \Sigma-\frac{1}{4 \pi} \iint_{\Sigma_{s} \cup \Sigma_{w}}\left[\mu \vec{\nabla}\left(\frac{1}{r}\right) \cdot \vec{n}\right] d \Sigma
$$

Ce dernier peut donc être généré par des distributions surfaciques de sources et de doublets normaux d'intensités respectives $\sigma$ et $\mu$. C'est l'intérêt majeur de la formulation intégrale qui ramène le problème de la détermination de la fonction $\varphi$ dans l'espace à celui de la détermination de deux fonctions $\sigma$ et $\mu$ sur une surface. En tenant compte de la condition de Dirichlet intérieur : $\varphi_{i}=0$ et en utilisant la notation introduite par Hadamard [3], on obtient :

$$
\frac{\mu(M)}{2}-\frac{1}{4 \pi} \iint_{\Sigma_{s} \cup \Sigma_{w}}\left[\mu \vec{\nabla}\left(\frac{1}{r}\right) \cdot \vec{n}\right] d \Sigma=\frac{1}{4 \pi} \iint_{\Sigma_{s}}\left[\sigma\left(\frac{1}{r}\right)\right] d \Sigma
$$

Il s'agit d'une équation de Fredholm de deuxième espèce dans laquelle le second membre est une fonction connue du fait de l'expression des conditions de glissement qui fournissent la valeur de la simple couche $\sigma$ sur le corps.

Compte-tenu de la condition sur le sillage et de la condition de Joukowski, la résolution du système engendré par la formule (7) ci-dessus va permettre de déterminer la densité superficielle de double couche $\mu$ à la surface du corps et sur le sillage dont la valeur est liée à celle du potentiel de perturbation extérieur par les relations :

$$
\begin{aligned}
& \begin{cases}\mu(M)=-\varphi(M) & \begin{array}{l}
\text { si le point } M \text { appartient à la sur- } \\
\text { face de l'aile } \Sigma_{s}
\end{array} \\
\mu(M)=\left(\varphi^{-}-\varphi^{+}\right)(M) & \begin{array}{l}
\text { si le point } M \text { appartient au } \\
\text { sillage }
\end{array}\end{cases} \\
& \text { II } \begin{array}{l}
\text { RÉSOLUTION. ASPECTS NUMÉRI- } \\
\text { QUES }
\end{array}
\end{aligned}
$$

\subsection{Discrétisation}

La résolution numérique du problème nécessite une discrétisation de la formulation intégrale exposée précédemment, ce qui nous amène, dans un premier temps, à discrétiser la structure étudiée. La demi-aile est divisée en 17 sections, chacune d'elles correspondant à une coupe suivant un plan parallèle au plan de symétrie de l'aile. Ces sections, réparties selon l'envergure à l'aide d'une loi en progression géométrique, définissent des bandes dont la largeur décroît en se rapprochant de l'extrémité de l'aile. Les bandes sont numérotées de 1 à 16 à partir de la section centrale située dans le plan de symétrie de l'aile. Chaque section étant homothé- 
tique de celle située dans le plan de symétrie, il suffit de mailler cette dernière pour obtenir, grâce à la connaissance de la loi d'évolution des cordes selon l'envergure, le maillage complet de l'aile. Il s'agit d'un profil NACA 16020 ( $20 \%$ d'épaisseur relative), non cambré, discrétisé en 40 facettes, soit 20 facettes à l'intrados et 20 facettes à l'extrados. L'envergure de l'aile et la corde à l'emplanture mesurent respectivement $360 \mathrm{~mm}$ et $120 \mathrm{~mm}$. L'aile étudiée a donc un allongement égal à 3,82 . Dans la suite de la présente étude, cette aile sera désignée sous le nom «aile A.C.C. ", ses caractéristiques ayant été fixées par les membres de l'A.C.C. L'aile est mise en incidence autour de l'axe parallèle à l'axe $O z$ et passant par le bord de fuite de sa section centrale.

L'influence de chaque panneau de l'aile et du sillage doit être calculée en chacun des points de contrôle de l'aile. Ces coefficients ne dépendant que de la géométrie de l'aile sont les coefficients d'influence. En discrétisant l'équation de Fredholm (7) obtenue précédemment, on transforme les intégrales en sommes, les valeurs de $\sigma$ et $\mu$ sur chaque panneau étant supposées constantes. La formule intégrale (7), valable en tout point de la surface continue de l'aile entière, est donc remplacée par une formule discrétisée, valable uniquement sur les $2 \mathrm{~N}$ panneaux que compte l'aile. Nous sommes donc en présence de $2 N$ inconnues puisque cette formule est écrite au point de contrôle de chaque panneau.

\subsection{Théorie des images. Simplifications}

La théorie des images, qui doit servir à modéliser les parois de la veine lors du placement de l'aileron en milieu confiné, est déjà appliquée au calcul de l'aile en milieu infini. L'écoulement étant symétrique par rapport au plan $(O x y)$, il suffit de l'étudier dans le domaine $z>0$. Mais il faut bien sûr tenir compte de l'influence de l'autre moitié de l'aile. Pour obtenir une influence nulle en vitesse normale sur le plan $(O x y)$, il faut placer les mêmes distributions de sources et de doublets normaux sur deux panneaux homologues. En réalité, nous sommes donc en présence de $N$ inconnues puisque ce sont les mêmes singularités de part et d'autre du plan de symétrie $z=0$.

Le principe des symétries, appliqué au calcul d'un écoulement bidimensionnel, a déjà fait l'objet de nombreuses études [2]. En revanche, à la connaissance des auteurs, son application à un écoulement tridimensionnel semble n'avoir

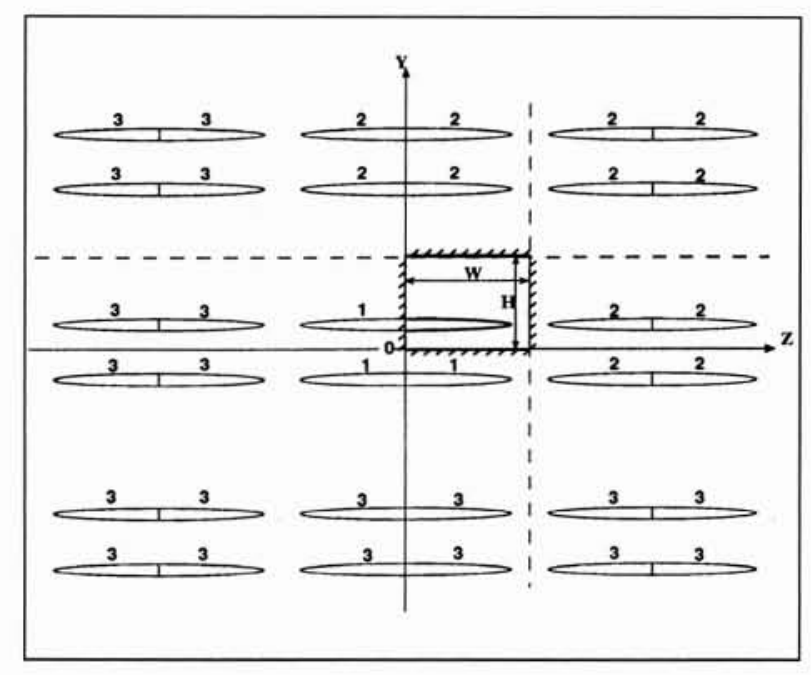

2. Principe de numérotation des symétries. jamais été mise en œuvre. Nous désignons par $H$ la hauteur de la veine, fixée à $175 \mathrm{~mm}$, et par $L$, la largeur de la veine, pouvant varier de $180 \mathrm{~mm}$ à $240 \mathrm{~mm}$.

La demi-aile en incidence est positionnée dans la veine d'étude en appuyant sa section 1, correspondant à l'emplanture, contre la paroi latérale gauche de la veine, dans le sens de l'écoulement. Afin de pouvoir faire varier le niveau de confinement, la veine a une section rectangulaire variable, la paroi verticale proche de l'extrémité de l'aile pouvant être déplacée transversalement. La distance comprise entre l'extrémité et la paroi, appelée entrefer, varie de 0 à l'infini.

Les quatre parois sont prises en compte simultanément en effectuant des symétries par rapport au fond, au plafond et aux deux parois latérales de la veine, conformément à la théorie des images (fig. 2). Comme dans le cas bidimensionnel, les différents ailerons engendrés par les symétries par rapport au fond et au plafond vont former une série infinie de période spatiale $2 H$. La première symétrie, effectuée par rapport au fond, génère un aileron; la seconde, par rapport au plafond, génère deux ailerons; la troisième, par rapport au fond, deux ailerons; et ainsi de suite. A chaque symétrie, les ailerons se déduisent de ceux générés par les deuxième et troisième symétries, uniquement par translation verticale de valeur $2 k H$ (avec $k$ entier positif ou négatif). Ce procédé permet de placer l'aileron entre deux plans horizontaux mais ne tient pas compte des parois latérales. Pour cela, nous effectuons également des symétries par rapport à ces parois, selon le même principe que celui utilisé précédemment dans le sens vertical, les ailerons engendrés se déduisant toujours de ceux générés par les deuxième et troisième symétries, mais cette fois par translation horizontale de valeur $2 \mathrm{~kL}$. Ces symétries ne s'effectuent pas seulement par rapport aux parois de la veine, mais par rapport aux plans entiers contenant ces parois. Le nombre $n$ de symétries correspond en réalité à $n$ symétries par rapport aux parois latérales et $n$ symétries par rapport au fond et au plafond. Ce nombre $\mathrm{n}$ est un paramètre du calcul, laissé au choix de l'utilisateur. Il doit cependant être impair, un nombre pair ayant tendance à décentrer l'aileron de base de l'ensemble des ailerons générés. Soit $N A(n)$ le nombre d'ailerons générés par $n$ symétries; nous avons: $N A=4 n^{2}$.

De nombreux tests de calcul nous ont permis de valider le modèle numérique basé sur le couplage méthode potentielle - théorie des images. Le code corps épais développé prend correctement en compte les effets de parois, les conditions de glissement étant parfaitement vérifiées sur les parois, sur lesquelles les vitesses sont purement tangentielles. Deux tourbillons voisins ont des sens de rotation opposés, créant ainsi un champ de vitesse contenu dans le plan médian, ce qui permet de matérialiser fictivement la paroi.

\subsection{Mode de résolution}

La technique de résolution consiste à résoudre le problème bande par bande. Cette méthode indirecte est stable et présente une bonne convergence. Afin de calculer les vecteurs inconnus $\mu(j, k)$ relatifs à l'ensemble des bandes (aile + sillage), nous utilisons une procédure itérative de résolution par blocs, du type Gauss-Seidel par blocs, chaque bloc correspondant à une bande de l'aile. L'algorithme utilisé sur les blocs est une méthode directe du type Householder, le calcul d'une nouvelle valeur $\mu(j, k)^{i}$ s'effectuant en supposant connus les vecteurs $\mu(j, k)^{i-1}$ obtenus à l'itération $(i-1)$.

La méthode potentielle utilisée ici pour modéliser l'écoulement [4] permet d'affecter une valeur de double couche constante à chaque bande de sillage, c'est-à-dire que les intensités de doublet normal de tous les panneaux d'une même bande de sillage sont identiques. Chaque élément de la matrice étant la somme des coefficients d'influence de deux panneaux symétriques par rapport au plan $z=0$, l'influence de la partie symétrique de l'aile est ainsi automa- 
tiquement prise en compte, sans augmenter le nombre total d'inconnues. Le recours à la formulation du champ lointain permet un gain de temps considérable. La matrice est à diagonale dominante, chaque valeur $1 / 2$ sur cette diagonale représentant l'auto-influence de chaque panneau sur la bande de l'aile considérée, c'est-à-dire son coefficient d'influence doublet sur lui-même. Au cours du processus itératif, la précision relative sur la valeur des doublets normaux est fixée à $10^{-3}$

\subsection{Modélisation du sillage}

Le sillage initial est matérialisé par une nappe fluide d'épaisseur nulle, composée de lignes tourbillons s'échappant du bord de fuite, sur lesquelles les doublets sont réévalués à chaque itération afin d'assurer la conservation de la circulation selon l'envergure pendant sa déformation. La géométrie du sillage peut être calculée de deux façons différentes : par relaxation ou par déformation pseudo-lagrangienne. Dans les deux cas, la vitesse moyenne est calculée en chaque nœud du sillage, son équilibre dynamique impliquant que chaque ligne vortex soit une ligne de courant. Le sillage est donc déformé par étapes successives, les conditions de type Dirichlet résolues à chaque pas permettant l'établissement de la nouvelle géométrie du sillage. Ce procédé itératif se poursuit jusqu'à la convergence, c'est-à-dire jusqu'à l'obtention de la forme définitive du sillage. Celle-ci est atteinte lorsque l'écart absolu maximal entre 2 déformées successives de la nappe devient inférieur à $1 \mathrm{~mm}$, soit moins de $1 \%$ de la corde à l'emplanture $(C=120 \mathrm{~mm})$. Dans le cas relaxé, pour chaque itération, la géométrie de chaque ligne vortex est recalculée dans son intégralité à partir du bord de fuite. Dans le cas lagrangien, le sillage est initialisé par une rangée de panneaux au bord de fuite. A chaque pas de temps, il est augmenté d'une rangée de panneaux supplémentaire. Cette procédure instationnaire est utilisée pour le cas particulier de l'écoulement relatif stationnaire. Le sillage proche converge vers sa forme finale en régime permanent. Notons au passage que ce procédé permet également de calculer la déformée de la nappe en régime instationnaire. Cette méthode s'avère être beaucoup plus performante que la méthode de déformation par relaxation.

\section{IV —VALIDATION. EXPLOITATION. RÉSULTATS}

\subsection{Prédiction du coefficient de portance}

Afin de compléter la validation du code, nous avons testé la capacité du modèle à prévoir la valeur du coefficient de portance pour une aile placée en milieu confiné. Aucune mesure expérimentale de la portance n'ayant été réalisée sur notre moyen d'essai dans le cadre de cette étude, nous avons choisi de comparer les résultats fournis par le code aux mesures réalisées au Laboratoire d'Hydrodynamique de l'Ecole Navale (L.H.E.N. - Brest) en reproduisant numériquement avec précision la configuration géométrique utilisée dans ce tunnel.

La veine d'essai a une section carrée de $192 \mathrm{~mm}$ de côté. L'aile utilisée a une corde de $80 \mathrm{~mm}$ et une demi-envergure de $120 \mathrm{~mm}$. Il s'agit donc d'une aile ayant les mêmes caractéristiques adimensionnelles - allongement, épaisseur relative - que l'aile A.C.C. décrite précédemment. L'aile est installée sur une balance hydrodynamique à 3 composantes qui permet de mesurer les efforts exercés sur l'aile (portance $[N]$, traînée $[N]$, couple de torsion $[m . N]$ ). La vitesse amont est égale à $7 \mathrm{~m} / \mathrm{s}$, ce qui nous donne un nombre de Reynolds de $5,5 \cdot 10^{5}$. La comparaison du coefficient de por-

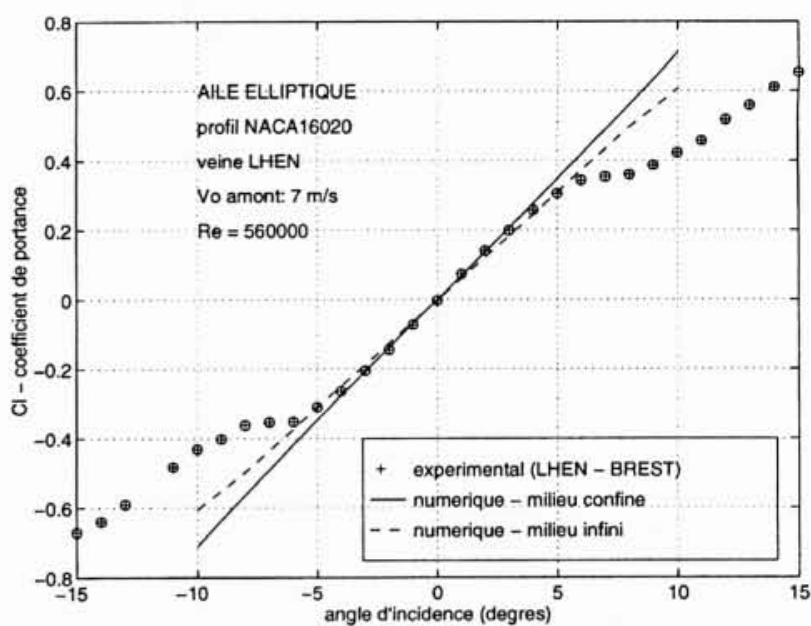

3. Comparaison des coefficients de portance obtenus numériquement et expérimentalement.

tance $C_{L}$ obtenu par le code avec les mesures expérimentales est présentée sur la figure 3 .

Le modèle donne une variation linéaire du $C_{L}$ en fonction de l'incidence. Pour les incidences inférieures à 5 degrés (zone linéaire), la correspondance est très satisfaisante, tandis que pour les incidences supérieures, le modèle se révèle incapable de prédire la baisse de portance observée expérimentalement. Cela est dû au fait qu'il ne prend pas en compte le décollement à la surface de l'aile. Afin de quantifier avec précision l'effet global du confinement dû aux parois de cette veine sur l'écoulement, nous avons effectué le calcul de l'écoulement autour de cette même aile, mais placée cette fois en milieu infini. La droite représentant l'évolution du coefficient de portance en fonction de l'incidence dans ce cas fictif, impossible à reproduire expérimentalement, est représentée en pointillés sur la figure 3.

La comparaison des pentes des deux droites met en évidence l'influence non négligeable sur l'écoulement des parois du tunnel du L.H.E.N. lors de la mesure des efforts. Les coefficients directeurs des deux droites correspondant au milieu infini et au cas confiné sont respectivement 0,06 et 0,07 . Pour une incidence de 4 degrés, cela se traduit par des coefficients de portance respectifs de 0,24 et 0,28 . La présence des parois du tunnel modifie donc de $13 \%$ le $C_{L}$, sa valeur en milieu infini étant prise comme référence. De cette analyse, il ressort que le code corps épais développé prédit correctement le coefficient de portance $C_{L}$, y compris en milieu confiné, à condition que l'incidence demeure inférieure à 5 degrés. A partir de cette remarque, nous pouvons donc observer l'effet du confinement sur les mesures réalisées dans le tunnel hydrodynamique du L.E.G.I. Pour cela, nous effectuons une comparaison des coefficients de portance obtenus par le modèle pour un entrefer variant de 60 à $5 \mathrm{~mm}$ (fig. 4).

Ces résultats mettent nettement en évidence le gain de portance obtenu en augmentant progressivement le niveau de confinement. Le tourbillon image induit une vitesse supplémentaire qui, en venant se composer avec la vitesse axiale, est équivalente à une augmentation d'incidence. L'augmentation de portance observée est donc essentiellement due à une augmentation d'incidence effective liée au rapprochement du tourbillon image. A l'inverse, plus l'entrefer augmente, plus la portance diminue, en tendant asymptotiquement vers une valeur limite lorsque l'entrefer devient très grand et tend vers l'infini. Le cas correspondant à un entrefer de $2000 \mathrm{~mm}$ est représenté en pointillés sur la figure 4 .

Dans le cadre de la présente étude, la hauteur de la veine demeure constante lors des modifications du niveau de confinement. Cela permet de mesurer correctement l'effet sur l'écoulement du déplacement de la paroi latérale et de le 


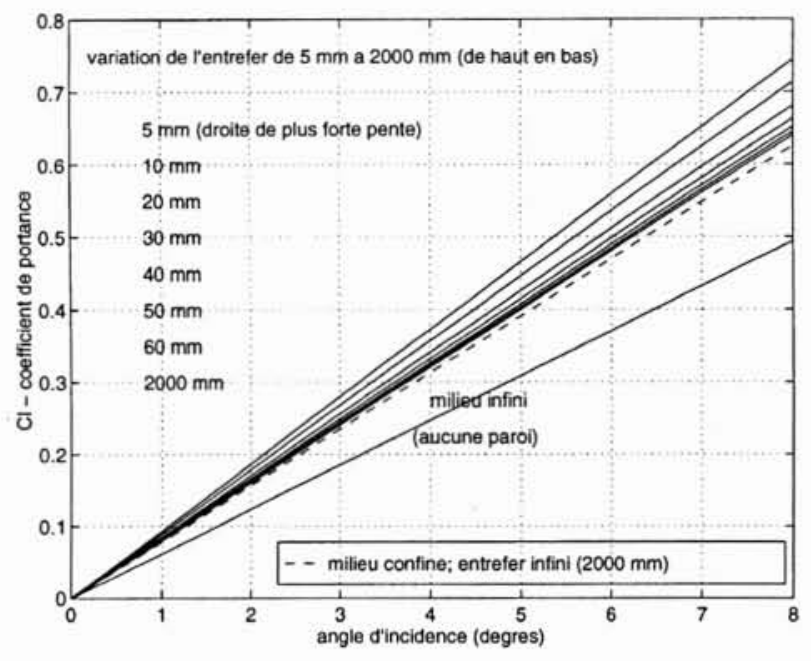

4. Influence du niveau de confinement sur la portance de l'aile.

découpler de l'effet des parois horizontales. Si ces dernières sont rapprochées de l'aile, la portance augmente considérablement. Dans le cas extrême, si les parois horizontales sont très proches de l'aile, l'écoulement est quasiment bloqué par l'aile qui occupe dans ce cas la majeure partie de la section de la veine.

Par abus de langage, le plus faible niveau de confinement réalisable expérimentalement, à savoir un entrefer maximal de $60 \mathrm{~mm}$ lorsque la paroi mobile est la plus éloignée de l'extrémité de l'aile, a été baptisé " milieu infini " lors des mesures expérimentales réalisées sur le tunnel hydrodynamique. La portance obtenue dans cette configuration dépasse de $2 \%$ la portance obtenue dans le cas d'un entrefer « infini » $(2000 \mathrm{~mm})$, laquelle dépasse de $27 \%$ celle obtenue en milieu infini, en l'absence de toute paroi. Ce dernier écart est en partie dû à l'entrefer infini du côté du bout de l'aile mais surtout à l'absence de deux parois: le fond et le plafond de la veine. En effet, leur absence réduit considérablement la différence de pression entre l'intrados et l'extrados de l'aile qui est à l'origine de la portance de l'aile. Cela est mis en évidence par l'important écart entre les $C_{L}$ obtenus lorsque l'entrefer est égal à $2000 \mathrm{~mm}$ et dans le cas du milieu infini, en l'absence de toute paroi. Notons que le cas fictif du milieu infini est difficilement réalisable expérimentalement. L'effet du fond et du plafond est donc bien découplé de l'effet de la paroi latérale mobile qui permet de modifier l'entrefer.

\subsection{Répartition de la circulation en envergure}

La circulation $\Gamma$ autour de chaque bande est obtenue par la formule :

$$
\Gamma=\sum_{i=1}^{N P(1)}\left(\vec{V}_{i} \cdot d \overrightarrow{1}_{i}\right)
$$

où $N P(1)$ représente le nombre de panneaux sur une bande

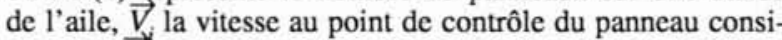
déré et $\overrightarrow{d 1_{i}^{\prime}}$ l'élément de longueur axial sur ce même panneau.

Sur la figure 5 sont présentées les courbes de circulation en envergure obtenues pour différents niveaux de confinement. Il s'agit de la circulation obtenue dans chaque section de l'aile. Elle est adimensionnalisée par la circulation $\Gamma 0$ à l'emplanture obtenue lorsque l'entrefer tend vers l'infini. Pour un entrefer de $2000 \mathrm{~mm}$, la circulation à l'emplanture

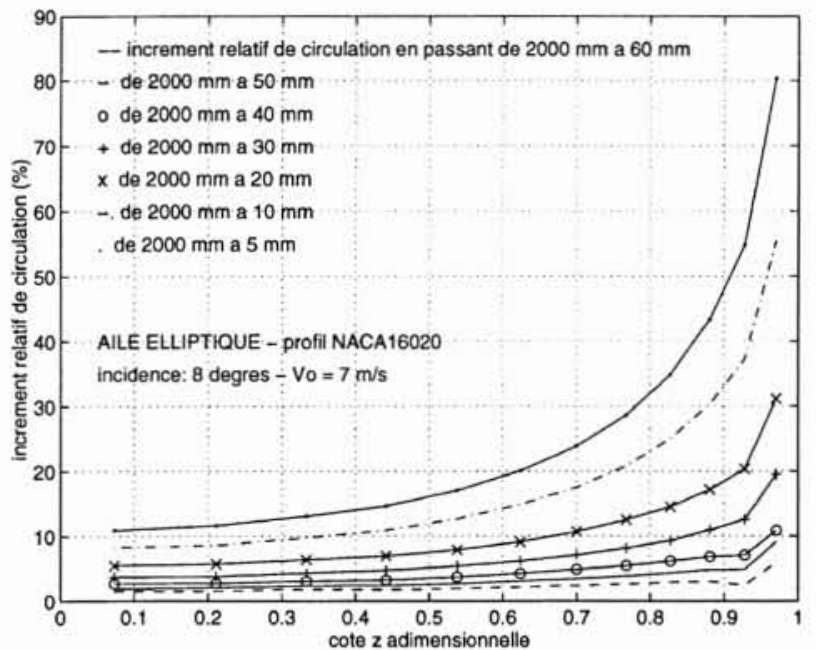

5. Influence du confinement sur la distribution de circulation en envergure.

Г0 est égale à $0,288 \mathrm{~m}^{2} / \mathrm{s}$ (incidence : 8 degrés, $V_{0}=7 \mathrm{~m} / \mathrm{s}$ ). Cette représentation permet de mettre en évidence le gain de circulation obtenu lorsque le niveau de confinement est augmenté progressivement. Ainsi, un entrefer de $5 \mathrm{~mm}$ conduit à une circulation à l'emplanture supérieure de $11 \%$ à celle obtenue lorsque l'entrefer est de $2000 \mathrm{~mm}$ et supérieure de $40 \%$ à celle obtenue en milieu infini. Remarquons tout d'abord la distribution quasi-elliptique de la circulation en envergure autour de l'aile, caractéristique de la théorie de la ligne portante qui prévoit une distribution de circulation elliptique pour une aile de forme en plan elliptique. Comme le coefficient de portance, la circulation autour de l'aile augmente avec le niveau de confinement. Inversement, lorsque l'entrefer varie de $5 \mathrm{~mm}$ à $60 \mathrm{~mm}$, la circulation autour de l'aile diminue et tend asymptotiquement vers une distribution correspondant au cas où l'entrefer est infini. Cette répartition est représentée en pointillés sur la figure 5 .

En faisant varier l'entrefer de $60 \mathrm{~mm}$ à $5 \mathrm{~mm}$, nous avons également constaté que l'incrément absolu de circulation demeure constant selon l'envergure. En valeur relative, l'augmentation de circulation est donc plus importante en

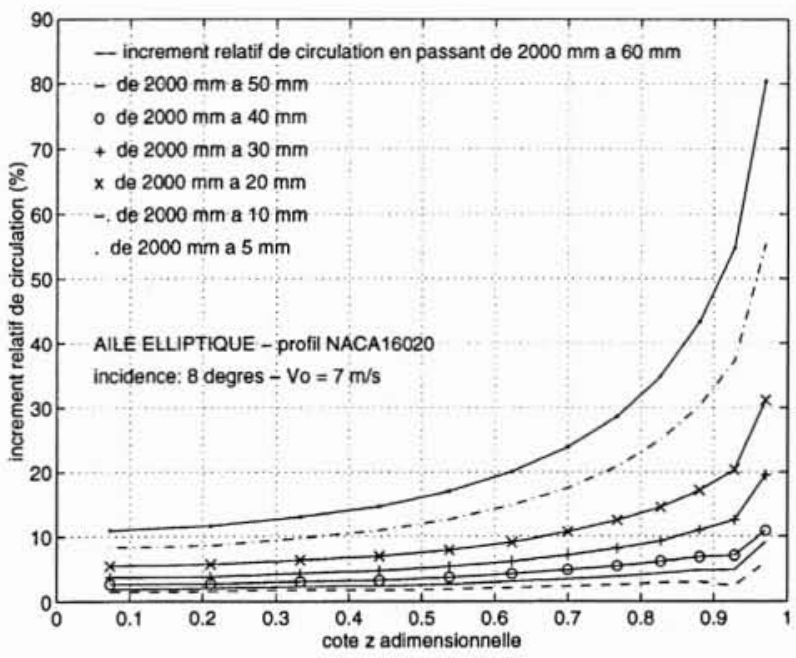

6. Evolution de l'incrément relatif de circulation en envergure en fonction du niveau de confinement. 
extrémité d'aile (fig. 6). L'incrément de circulation entre deux configurations géométriques successives est d'ailleurs d'autant plus important que le niveau de confinement est élevé. Cette remarque trouve son explication dans l'augmentation de l'incidence induite selon l'envergure lorsque le tourbillon image se rapproche du tourbillon étudié.

\section{$\mathrm{V} \square$ CONCLUSION}

Les nombreuses comparaisons entre les résultats obtenus par le code corps épais et les mesures expérimentales mettent en évidence les performances du modèle numérique développé. Une correspondance très satisfaisante entre résultats numériques et mesures expérimentales a été observée. Le modèle se révèle donc être un prédicteur fiable des caractéristiques de l'écoulement tridimensionnel subcavitant autour de l'aile étudiée en régime permanent. L'influence du niveau de confinement sur l'écoulement a été mise en évidence d'une manière globale, par analyse du coefficient de portance et de la distribution de circulation selon l'envergure de l'aile.

La vaste exploitation des résultats fournis par le code permet d'aboutir aux conclusions suivantes :

- D'un point de vue global, le coefficient de portance est obtenu avec précision dans son domaine de variation linéaire, c'est-à-dire pour des incidences inférieures à 6 degrés pour l'aile considérée. Le code singularités 3D est donc un outil précieux pour caractériser l'effet de confinement inhérent à la géométrie de la section d'un tunnel hydrodynamique, à condition que l'incidence de l'aile ne soit pas trop élevée. Au-delà de 6 degrés, le décollement de l'écoulement qui se produit à l'extrados de l'aile entraîne une chute de portance, non prévisible par ce type de code.

- La dernière ligne vortex est assimilée à la ligne moyenne du tourbillon marginal [2]. La circulation mesurée autour du tourbillon marginal est caractéristique de l'intensité tourbillonnaire du tourbillon et est directement reliée à la circulation à l'emplanture de l'aile.

- Plus le niveau de confinement augmente, moins le tourbillon marginal est dévié vers l'emplanture [2], plus l'enroulement tourbillonnaire devient axisymétrique [2], et plus les circulations autour de l'aile et du tourbillon augmentent, entraînant une augmentation de la portance de l'aile.

Les nombreux résultats obtenus par cette approche témoignent de l'adaptabilité de la méthode intégrale aux problèmes d'hydrodynamique et met en évidence ses qualités de précision, de rapidité et de souplesse.

\section{RÉFÉRENCES}

[1] Boulon O., 1996. - « Etude expérimentale de la cavitation de tourbillon marginal. Effets instationnaires, de germes et de confinement ", Thèse de Doctorat de 1'Institut National Polytechnique de Grenoble.

[2] Deniset F., 1996. - « Modélisation numérique des conditions d'apparition de la cavitation de tourbillon marginal sur une aile-3D. Effet de confinement. ", Thèse de Doctorat de 1'Institut National Polytechnique de Grenoble.

[3] Hadamard J., 1923. - « Lectures on Cauchy's problem in linear partial differential equations ", New Heaven, Yale University Press.

[4] Maître T., 1988. — « Modélisation de l'écoulement autour d'une hélice marine par la méthode du potentiel ", Thèse de Docteur-Ingénieur, Institut National Polytechnique de Grenoble. 